\title{
Enhanced Epidermal Growth Factor, Hepatocyte Growth Factor, and Vascular Endothelial Growth Factor Expression in Tuberous Sclerosis Complex
}

\author{
Whitney E. Parker, ${ }^{*}$ Ksenia A. Orlova, ${ }^{*}$ \\ Gregory G. Heuer, ${ }^{\dagger}$ Marianna Baybis, ${ }^{*}$ \\ Eleonora Aronica, ${ }^{\ddagger}$ Michael Frost, ${ }^{\S}$ \\ Michael Wong, ${ }^{\text {ๆ }}$ and Peter B. Crino*
}

\begin{abstract}
From the PENN Epilepsy Center and Departments of Neurology" and Neurosurgery, ${ }^{\dagger}$ University of Pennsylvania Medical Center, Philadelphia, Pennsylvania; the Netherlands Institute for Neuroscience and Department of (Neuro)Pathology, ${ }^{\ddagger}$ Academic Medical Center, University of Amsterdam, The Netherlands; the Epilepsy Institute in the Netherlands Foundation (Stichting Epilepsie Instellingen Nederland, SEIN), Heemstede, The Netherlands; the Minnesota Epilepsy Group, ${ }^{\S}$ St. Paul, Minnesota; and the Department of Neurology," Washington University, St. Louis, Missouri
\end{abstract}

Epidermal growth factor (EGF), hepatocyte growth factor (HGF), and vascular endothelial growth factor (VEGF) regulate angiogenesis and cell growth in the developing brain. EGF, HGF, and VEGF modulate the activity of the mammalian target of rapamycin (mTOR) cascade, a pathway regulating cell growth that is aberrantly activated in tuberous sclerosis complex (TSC). We hypothesized that expression of EGF, HGF, VEGF, and their receptors EGFR, c-Met, and Flt-1, respectively, would be altered in TSC. We show by cDNA array and immunohistochemical analysis that EGF, EGFR, HGF, c-Met, and VEGF, but not Flt-1, mRNA, and protein expression was up-regulated in Tsc1 conditional knockout (Tsc1 ${ }^{\text {GFAP }}$ CKO) mouse cortex. Importantly, these alterations closely predicted enhanced expression of these proteins in tuber and subependymal giant cell astrocytoma (SEGA) specimens in TSC. Expression of EGF, EGFR, HGF, c-Met, and VEGF protein, as well as hypoxia inducible factor$1 \alpha$, a transcription factor that regulates VEGF levels and is also modulated by mTOR cascade activity, was enhanced in SEGAs $(n=6)$ and tubers $(n=10)$ from 15 TSC patients. Enhanced expression of these growth factors and growth factor receptors in human SEGAs and tubers and in the Tsc1 ${ }^{\text {GFAP }}$ CKO mouse may account for enhanced cellular growth and proliferation in tubers and SEGAs and provides potential target molecules for therapeutic development in TSC. (Am J Pathol 2011, 178:296-305; DOI: 10.1016/j.ajpath.2010.11.031)

Tuberous sclerosis complex (TSC) is an autosomal dominant disorder that results from mutations in the TSC1 or TSC2 genes, which encode TSC1 and TSC2 proteins, respectively. ${ }^{1,2}$ Many individuals with TSC exhibit cognitive disability and autism, ${ }^{3}$ and more than $75 \%$ of TSC patients develop seizures. ${ }^{4-6}$ Examination of the brain demonstrates cortical tubers and subependymal nodules (SENs) in more than $70 \%$ of TSC patients. Tubers are developmental malformations of the cerebral cortex highly associated with epilepsy and neurocognitive abnormalities. SENs are nodular lesions (typically smaller than $1 \mathrm{~cm}$ ) located on the surfaces of the lateral and third ventricles. In approximately $10 \%$ to $20 \%$ of TSC patients, subependymal giant cell astrocytomas (SEGAs) arise within the lateral ventricles, often near the foramen of Monro. SEGAs are World Health Organization grade I tumors with low mitotic index as evidenced by Ki-67 immunoreactivity suggestive of slow cellular proliferation. ${ }^{7,8}$ It is widely believed that SENs grow to form SEGAs, although the molecular mechanisms governing transformation from SEN to SEGA are unknown. ${ }^{9}$ Both SENs and SEGAs consist of dysmorphic glial cells, enlarged giant cells (GCs), and spindle-shaped cells of unknown phenotype. ${ }^{10,11}$ Cellular immunoreactivity for glial fibrillary acidic protein (GFAP), neurofilament, S-100, neuron-specific eno-

Supported by NS045877, NS045021, and Department of Defense CDMRP-TSC Program grants (P.B.C.); the National Epilepsy Fund "Power of the Small," Hersenstichting Nederland (NEF 02-10 and NEF 05-11) and Stichting Michelle (M06.011) (E.A.); and AOA and AANS medical student research awards (W.E.P.).

Accepted for publication September 21, 2010.

Supplemental material for this article can be found at http://ajp. amjpathol.org or at doi:10.1016/j.ajpath.2010.11.031.

Address reprint requests to Peter B. Crino, M.D., Ph.D., Department of Neurology, PENN Epilepsy Center, University of Pennsylvania Medical Center, 3 West Gates Bldg., 3400 Spruce St., Philadelphia, PA 19104. E-mail: peter.crino@uphs.upenn.edu. 
lase, and synaptophysin proteins suggests that SEGAs contain both glial and neuronal cell types. Lineage studies have demonstrated that SEGAs express cellular markers found in progenitors derived from the subventricular zone adjacent to the lateral ventricles ${ }^{12,13}$ and that many of these markers are also expressed in cortical tubers.

The TSC1 and TSC2 proteins combine to form a heterodimer that functions as an upstream modulator of the mammalian target of rapamycin (mTOR) pathway, which integrates growth factor and energy level signals to promote several cellular processes, including cell growth and proliferation, ${ }^{14}$ protein translation, and angiogenesis. TSC1 and TSC2 mutations are associated with loss of inhibitory modulation and consequent constitutive activation of the mTOR cascade, resulting in enhanced cell size and proliferation, especially under conditions favoring cell growth. Previous studies have suggested that altered growth factor expression may be associated with abnormal cellular architecture in the brains of TSC patients. For example, differential expression of neurotrophins and their receptors has been observed in cortical tubers, ${ }^{15}$ and TSC1-TSC2 mediated control of MTOR is modulated by insulin-like growth factor-1 (IGF-1). ${ }^{16-18}$ Recent evidence also suggests that mTOR signaling is regulated by several growth factors, such as epidermal growth factor (EGF) and hepatocyte growth factor (HGF), and that TSC1TSC2 may regulate downstream expression of select angiogenic factors, such as vascular endothelial growth factor (VEGF), via hypoxia inducible factor- $1 \alpha(\mathrm{HIF}-1 \alpha) .{ }^{19}$ For example, EGF regulates smooth muscle cell proliferation via its receptor EGFR through mTOR signaling. ${ }^{20}$ VEGF expression is up-regulated in the Eker rat TSC model, in mouse embryonic fibroblasts lacking Tsc2, and in facial angiofibromas from TSC patients. ${ }^{19,21,22}$ Brain and kidney lesions in TSC exhibit abnormally enhanced expression of the vascular endothelium protein marker CD31. ${ }^{23}$ Altered VEGF isoform $D$ levels were observed in serum from TSC patients with lymphangioleiomyomatosis. ${ }^{24}$ Of note, enhanced VEGF expression in TSC may occur via both mTOR-dependent and mTOR-independent mechanisms. ${ }^{25}$

Altered expression of EGF, HGF, VEGF, and their receptors EGFR, c-Met, and Flt-1 (VEGFR1) has not been investigated in the brain in TSC, yet considerable evidence suggests that these factors play a pivotal role in the mTOR cascade's influence on cellular phenotype and could therefore provide insight into the mechanism of TSC cortical pathogenesis. Thus, we assayed both mRNA and protein expression in the TSC1 conditional knockout (Tsc1GFAPCKO) mouse and in human TSC brain tissue specimens as a strategy to identify growth factors that could be targeted for therapeutic development in TSC.

\section{Materials and Methods}

\section{Tsc $1^{\text {GFAP }}$ CKO Mice}

Tsc1 ${ }^{\text {GFAP }}$ CKO mice were generated as previously described $^{26}$ at the Washington University School of Medicine in accordance with the guidelines established by the Animal Studies Committee of Washington University.
Samples at postnatal days 1 and 10 were analyzed for several reasons. First, at these time points, the active phases of embryonic neuronal migration are completed; thus, there is homeostatic expression of growth factors and receptors. Second, by postnatal days 1 and 10 there is active expression of the Cre transgene in the brain; thus, adequate knockout of Tsc1 could be documented. Third, we wanted to be certain that none of the altered growth factor expression was a consequence of early seizures and altered behavioral phenotypes that occur in these animals by postnatal day 20 .

\section{Extraction of mRNA from Cerebral Cortex in the Tsc $1^{\text {GFAP }}{ }^{2}$ KO Mice}

Poly $(A)$ mRNA was extracted from the cerebral cortex of Tsc $1^{\text {GFAP } C K O}$ or wild-type (Wt) mice ( $n=5$ each) as described previously. ${ }^{15}$ The entire cortex from both hemispheres was removed from the subcortical structures using a microscalpel at the level of the midhippocampus. Poly(A) mRNA served as a template for in vitro cDNA synthesis with avian myeloblastosis virus reverse transcriptase, and then double-stranded template cDNA was synthesized with T4 DNA polymerase I (BoehringerMannheim) from extracted cDNA. mRNA was amplified from the double-stranded cDNA with T7 RNA polymerase (Epicenter Technologies) incorporating ${ }^{32} \mathrm{P}$ CTP as a radiolabel. Amplified mRNA served as a template for a second round of cDNA synthesis with avian myeloblastosis virus reverse transcriptase, deoxynucleotide triphosphates, and N6 random hexamers (Boehringer-Mannheim). cDNA generated from amplified mRNA was made double stranded and served as template for a second mRNA amplification, again incorporating ${ }^{32} \mathrm{P}$ CTP radiolabel. The radiolabeled, amplified mRNA was used as a probe for cDNA arrays.

\section{cDNA Array Analysis}

cDNA arrays containing full-length mouse EGF, EGFR, HGF, c-Met, VEGF, and Flt-1 cDNAs were probed with ${ }^{32} \mathrm{P}$ CTP-radiolabeled mRNA amplified from the cortex (one probe per array). All hybridization reactions were performed twice for each probe. Glyceraldehyde-3-phosphate dehydrogenase cDNA was included to serve as a positive hybridization control, and pBlueScript plasmid cDNAs were used to define background levels of hybridization on each array. Prehybridization (8 hours) and hybridization (24 hours) conditions were in 6× SSPE buffer, $5 \times$ Denhardt's solution, 50\% formamide, 0.1\% SDS, and salmon sperm DNA, $200 \mu \mathrm{g} / \mathrm{ml}$, at $42^{\circ} \mathrm{C}$. Blots were washed in $2 \times$ standard saline citrate. mRNA probe hybridization to array cDNAs was determined by phosphorimaging and densitometry.

\section{Human Tissue Specimens}

Tuber and SEGA samples ( $n=15$ patients; seven females; 10 tubers; six SEGAs; mean age, 9.9 years; in one patient a tuber and SEGA were removed en bloc; Table 1) 
Table 1. Patient Demographics

\begin{tabular}{lclc}
\hline Sample & Age, years & Location & Genotype \\
\hline Tuber & 4 & Frontal & TSC1 \\
Tuber & 9 & Frontal & TSC2 \\
Tuber & 3 & Temporal & TSC2 \\
Tuber & 9 & Frontal & TSC2 \\
Tuber & 3 & Temporal & TSC1 \\
Tuber & 2 & Temporal & TSC1 \\
Tuber & 4 & Frontal & NMI \\
Tuber & 6 & Temporal & TSC2 \\
Tuber & 7 & Frontal & NMI \\
Tuber & 10 & Frontal & TSC2 \\
SEGA* & 10 & Lat vent & TSC2 \\
SEGA & 14 & Lat vent & TSC1 \\
SEGA & 21 & Lat vent & TSC2 \\
SEGA & 22 & Lat vent & TSC1 \\
SEGA & 19 & Lat vent & TSC2 \\
SEGA & 16 & Lat vent & NMI \\
SEN & 27 & Lat vent & TSC2 \\
SEN & 37 & Lat vent & TSC2 \\
SEN & 41 & Lat vent & TSC2 \\
Epilepsy control & 6 & Temporal & NMI \\
Epilepsy control & 14 & Temporal & NMI \\
Epilepsy control & 12 & Temporal & NMI \\
Epilepsy control & 9 & Temporal & NMI \\
Epilepsy control & 16 & Temporal & NMI \\
Control & 4 & Frontal & NMI \\
Control & 9 & Frontal & NMl \\
Control & 11 & Temporal & NMI \\
Control & 11 & Frontal & NMI \\
\hline
\end{tabular}

Table depicts age at time of surgery, lobar location of resection, and TSC genotype.

Lat vent, lateral ventricle; NMI, no mutation identified.

*Specimen contained both SEGA and cortical tuber.

were obtained from patients with clinically diagnosed TSC. There were no significant differences in age distribution in the TSC patient specimens used in the study. Tubers were removed as part of surgery for the treatment of intractable epilepsy, and SEGAs were removed to alleviate symptomatic hydrocephalus. Surgical tissue specimens were obtained from the Academic Medical Center, University of Amsterdam, Amsterdam, The Netherlands, and Minnesota Epilepsy Group, St. Paul, MN. Surgical localization of the tuber resection site reflected the seizure focus as determined by scalp or intracranial electroencephalographic monitoring. SEGAs were identified preoperatively by magnetic resonance imaging as non-neurologic, progressively enlarging lesions that exhibited enhancement with gadolinium. Three SENs were obtained post mortem from three TSC patients (Table 1) who died of nonneurologic causes. In these three specimens, morphologically normal cortex adjacent to histologically defined tubers was also obtained. Clinical mutation testing (indicated as "genotype" in Table 1) results among the tuber, SEN, and SEGA patients revealed a TSC1 mutation in five patients and a TSC2 mutation in 10 patients; mutation data were unavailable from three patients (indicated as "NMI" in Table 1).

Postmortem control brain tissue specimens ( $n=4$; two females; Table 1) were procured from the Brain and Tissue Bank for Developmental Disorders, University of Maryland (Baltimore, MD), from individuals who died of nonneurologic causes. Seizures were not terminal events in these patients, and none had a personal or family history of epilepsy or TSC. The cytoarchitecture of these specimens was intact. Additional surgical epilepsy control tissue consisted of temporal neocortical specimens ( $n=5$; three females; Table 1) obtained from individuals undergoing temporal lobectomy for intractable complex partial seizures (University of Pennsylvania Medical Center). These patients had no history or clinical findings compatible with a diagnosis of TSC, and the histologic features of the tissue samples were intact (these specimens were classified as epilepsy controls). All human tissue was obtained in accordance with protocols approved by the University of Pennsylvania Institutional Review Board and Committee on Human Research.

\section{Immunohistochemistry}

All mouse and human tissue samples were immersion fixed in $4 \%$ paraformaldehyde, embedded in paraffin, and sectioned at $8 \mu \mathrm{m}$. All fixed tissue blocks were hydrated through graded ethanols. Slides were pretreated with antigen unmasking solution (Vector Labs, Burlingame, CA) before immunostaining reactions. Sections were probed with one of the following antibodies: EGF (1:1000 dilution, rabbit polyclonal; Santa Cruz, Burlingame, CA), EGFR (1:100, rat polyclonal; Santa Cruz), EGFRvIII variant (courtesy of D. O'Rourke, M.D., Department of Neurosurgery, University of Pennsylvania, Philadelphia, PA), phospho-EGFR (phospho Y1068, 1:250, rabbit monoclonal; Abcam, Cambridge, MA), HGF (1: 500, mouse monoclonal; Abcam), c-Met (1:10, rabbit polyclonal; Abcam), VEGF (1:50, mouse monoclonal; Abcam), Flt-1 (1:100, mouse monoclonal; Santa Cruz), HIF-1 $\alpha$ (1:1500, mouse monoclonal; Abcam), phospho-S6 ribosomal protein (p-S6, Ser235/236; 1:100, rabbit polyclonal; Cell Signaling, New England Biolabs, Beverly, MA), or S6 ribosomal protein (detects endogenous S6 expression, including both phosphorylated and nonphosphorylated isoforms, 1:100, rabbit monoclonal; Cell Signaling) overnight at $4^{\circ} \mathrm{C}$ and with secondary antibodies at room temperature for 1 hour. The slides were visualized using avidin-biotin conjugation (Vectastain ABC Elite; Vector Labs) with 3,3'-diaminobenzidine. After immunolabeling, sections were dehydrated through graded ethanols and xylene and coverslip mounted (Permount).

\section{Quantitative Cell Counts}

Three representative contiguous digital photographs were obtained (20x magnification) from each mouse brain tissue section using image acquisition and analysis software (Spot RT CCD camera; Diagnostic Instruments Inc.; and Phase 3 Imaging System integrated with Image Pro Plus; Media Cybernetics, Silver Spring, MD). The three images spanned a $1-\mathrm{mm}^{2}$ region of interest (ROI) within the lateral neocortex that was operationally defined and standardized across all cortex specimens as dorsolateral cerebral cortex at the level of the rostral hippocampus midway between the superior sagittal sulcus and the rhinal sulcus (Bregma coordinate $-1.70 \mathrm{~mm}$ ). The area of 
the cortex for each ROI was determined with a glass micrometer under light microscopy.

In the human specimens, we were particularly interested in the number of GCs that expressed each protein growth factor marker. Thus, GCs were defined using maximal cell diameter based on cresyl violet and H\&E staining for quantitative cell counting analysis. Representative digital photographs were obtained (magnification, $\times 20$ ) under light microscopy from each tissue section ( $n=3$ sections per case) using image acquisition and analysis software as above. Each image spanned a $1-\mathrm{cm}^{2} \mathrm{ROI}$. Before final assignment as a GC by the software, each ROI was visually inspected and cellular elements erroneously included in the computerized analysis were deleted. Mean maximal diameter (cell diameter at its largest aspect) was calculated using Image Pro Plus software as expressed in pixel units that were converted to microns by direct calibration with a micrometer.

The relative optical density ratio (ODR) of labeled cells was calculated using Image Pro Plus software using a previously defined approach. ${ }^{15}$ The ODR is calculated by determining the level of pixel staining density in labeled cells versus the pixel density of the noncellular background (the cell densities are digitally subtracted from the image). An ODR greater than 3 was used as a threshold to define immunopositivity for a given antibody. In the mouse, the total numbers of p-S6-immunolabeled cells were determined for each case and then expressed as a

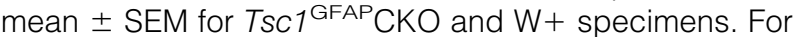
growth factors, we used a semiquantitative scale (0 indicating no staining to ++++ indicating intense labeling across most cells in each $\mathrm{ROI}$ ) to represent labeling density in each ROI. The size of p-S6-immunolabeled cells was defined using maximum cell diameter and determining the mean \pm SEM). In the human specimens, the total numbers of morphologically identified GCs were determined in each $\mathrm{ROI}$ for each case, and the mean \pm SEM numbers of GCs in ROls were determined across all 10 tubers and 6 SEGAs. Statistically significant differences in GCs expressing individual protein markers were determined by Student's $t$-test $(P<0.05)$.

\section{Western Analysis}

Lysates of Wt and Tsc1 ${ }^{\text {GFAPC }}$ CKO cortex were analyzed for p-S6 protein levels. A DuPont Kinetic Microplate Reader was used to approximate $15 \mu \mathrm{g}$ of total protein for each of the samples, which were individually loaded into separate wells of a $4 \%$ to $15 \%$ Tris- $\mathrm{HCl}$ polyacrylamide gel (Bio-Rad Laboratories) and electrophoresed at $60 \mathrm{~V}$. Proteins were then transferred overnight at $4^{\circ} \mathrm{C}$ onto a polyvinylidene difluoride (Millipore) membrane. Membranes were incubated in a 5\% nonfat dry milk blocking solution for 1 hour at room temperature (RT) and then probed with rabbit anti-p-S6 ribosomal protein (Ser235/236; 1:1000, overnight at $4^{\circ} \mathrm{C}$; Cell Signaling) antibodies. Rabbit antiglyceraldehyde-3-phosphate dehydrogenase (1:1000, 1 hour at RT; Cell Signaling) and rabbit anti- $\beta$-actin (1:1000, 1 hour at RT; Cell Signaling) served as protein loading controls. Membranes were then incubated for 1 hour at RT with horseradish peroxidase-conjugated donkey anti-rabbit IgG (1:3000; GE Health Care). Membranes were washed and developed using either electrochemiluminescence or electrochemiluminescence plus Western blotting detection reagents (Amersham, GE Health Care) as needed for horseradish peroxidase visualization.

\section{Statistical Analysis}

The expression of each mRNA was determined by analysis of the radiolabeled mRNA-cDNA hybridization intensity on each array using ImageQuant5.0 software. Nonspecific hybridization to pBlueScript plasmid cDNA was subtracted from the hybridization intensity of each mRNA-cDNA to define specific hybridization intensity. The relative hybridization intensity for each mRNA was determined by averaging the phosphorimaging density of all of the mRNA-cDNA hybrids on each individual array and then expressing each $\mathrm{mR}$ NA-cDNA hybrid as a percentage of the average hybridization intensity of the entire array. Differences in relative mRNA abundance were determined using a one-way analysis of variance, and a Bonferroni post hoc correction was applied to each univariate analysis
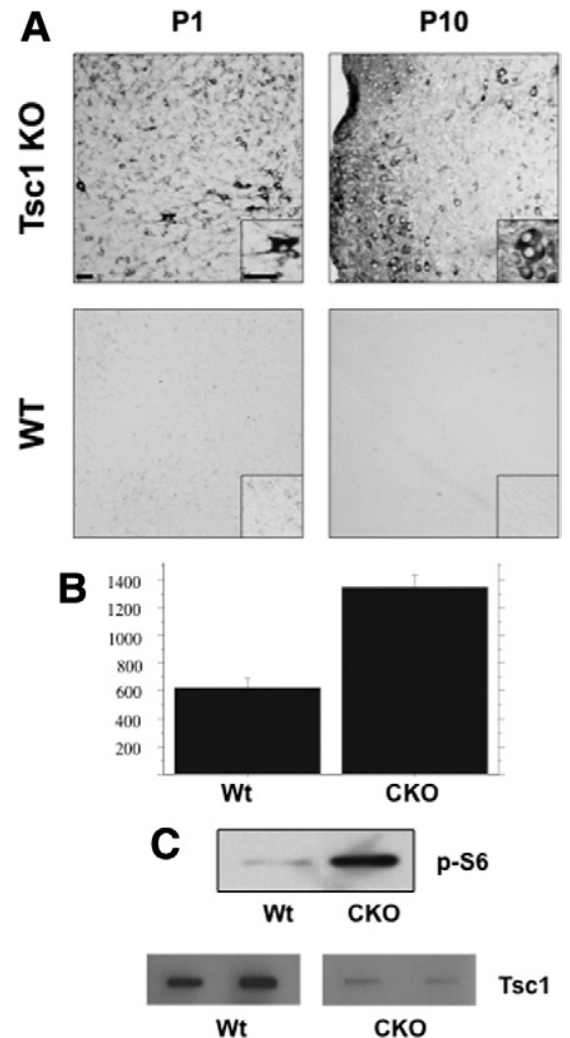

Figure 1. Enhanced $\mathrm{S} 6$ phosphorylation (p-S6) in Tsc1 ${ }^{\mathrm{GFAP}} \mathrm{CKO}$ mouse cortex A: At postnatal (P) days 1 and $10, T_{s c} 1^{\text {GFAP }}$ CKO (Tsc1 KO) cortex shows increased p-S6 expression compared with Wt cortex. Photograph taken at the level of the perirhinal sulcus; inset, original magnification, $\times 40$; individual cel labeling with p-S6 at postnatal days 1 and 10 . Scale bars, $20 \mu \mathrm{m}$. B: Graph showing quantitative cell counts of p-S6-labeled cells in WT and Tsc ${ }^{\text {GFAP }}{ }^{\text {CKO }}$ (CKO) mice at postnatal day 10. Note increased numbers of p-S6-labeled cells in CKO. C: Western blot depicting increased p-S6 levels in TsC $1^{\mathrm{GFAP}} \mathrm{CKO}$ versus Wt mouse cortex homogenates and cDNA array hybridization depicting reduced Tsc1 mRNA expression in CKO versus Wt mice. 


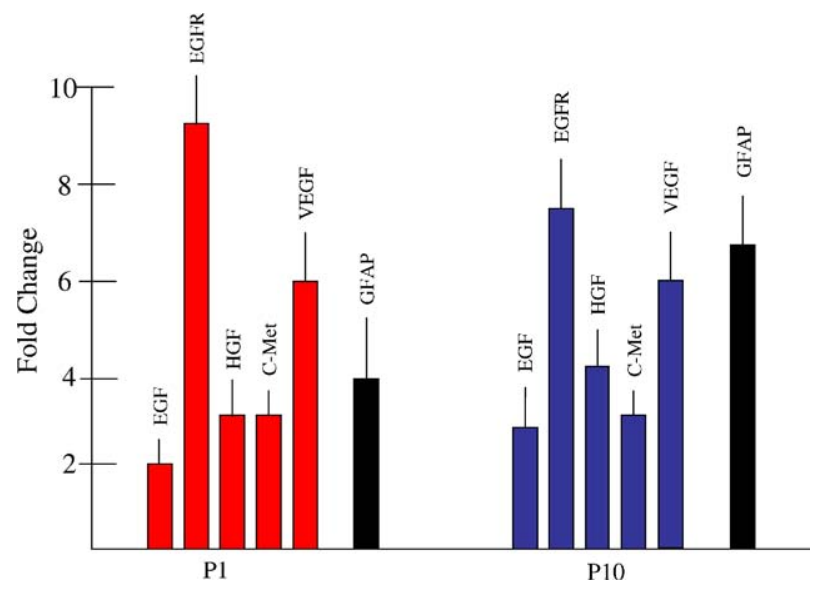

Figure 2. Increased expression of EGF, EGFR, HGF, c-Met, and VEGF mRNAs in Tsc1 $1^{\mathrm{GFAP}} \mathrm{CKO}$ versus Wt mouse cortex at postnatal days 1 (red) and 10 (blue). Bars represent mean change in relative abundance expressed as fold change relative to Wt cortex (lines above bars depict SEM; all significant $P<0.05$ ). Increased expression of GFAP mRNA at each time point is depicted in black.

of variance. If a significant difference was found with a Bonferroni-adjusted analysis of variance, individual post hoc comparisons were made using the Fisher's test $(P<0.05$ was considered significant).

\section{Results}

\section{Enhanced S6 Phosphorylation in the Tsc 1 GFAP CKO Mouse Cortex and Human TSC Brain Tissue}

The expression level of Tsc1 mRNA was determined at postnatal days 1 and 10 in the cerebral cortex in Tsc $1{ }^{\text {GFAP }}$ CKO mice. At postnatal day 1 , Tsc1 mRNA levels were reduced by $86 \% \pm 4 \%$, and at postnatal day 10 , levels were reduced by $92 \% \pm 7 \%$ compared with $\mathrm{Wt}$ mice $(n=10$ sections each in TSC1 GFAPCKO and Wt control samples at each time point, $P<0.05$; Figure $1 C$ ). Expression of Tsc2 mRNA at postnatal days 1 and 10 in cortex from the TSC1 GFAPCKO mice did not differ from that observed in Wt mice.

There was a low level of baseline p-S6 protein expression in cortical neurons and astrocytes of control Wt mice at postnatal days 1 and 10. p-S6-labeled astrocytes were observed throughout all cortical layers. In neurons,
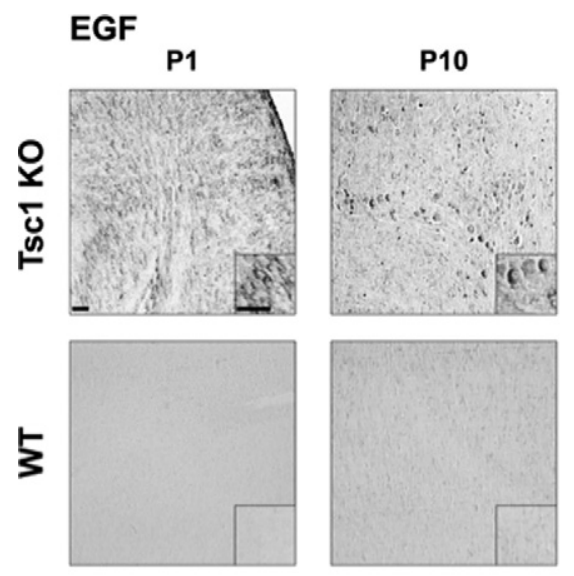

EGFR

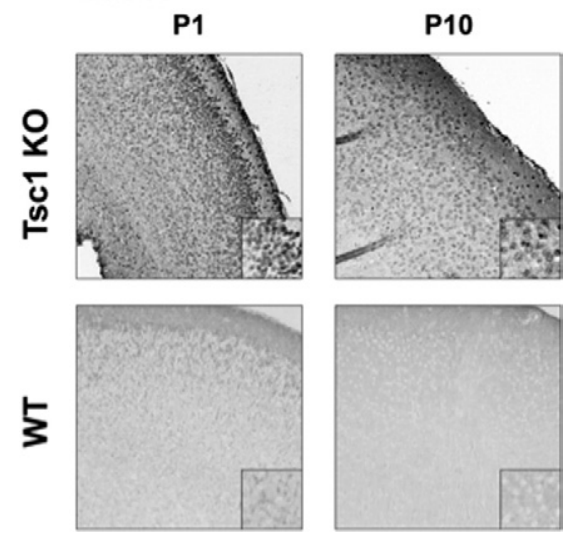

Figure 3. Enhanced expression of EGF and EGFR proteins in Tsc1 ${ }^{\mathrm{GFAP}} \mathrm{CKO}$ (Tsc1 KO) mouse versus WT mouse cortex at postnatal (P) days 1 and 10 inset, original magnification, $\times 40$. Note EGF expression in heterotopic cells in white matter at P10. Scale bars $20=\mu \mathrm{m}$.

p-S6 expression was observed primarily within the somatic and dendritic cytoplasm of pyramidal cells in layers $\mathrm{III}$ and V. At both postnatal days 1 and 10, there was a clear increase in the number of p-S6-labeled cortical cells in the TSC1 ${ }^{\text {GFAPC}}$ CKO mouse brain compared with control Wt brains (Figure 1A). Quantitative cell counts of p-S6-labeled cells were performed at postnatal days 1 and 10 , which antedates the onset of clinical seizures in these mice. There was a significant increase in the number of p-S6-labeled cells at postnatal day 1 (115 WT control and 336 TsC 1 GFAPCKO mice, $P<0.05$ ) and postnatal day 10 (665 control and 1319 Tsc ${ }^{\text {GFAP }}$ CKO mice, $P<0.05$ ) (Figure 1B). Western assay revealed markedly

Table 2. Laminar Expression of Growth Factors in Wt Versus $T s c 1^{\mathrm{GFAP}} \mathrm{CKO}$ Mice at Postnatal Day 10

\begin{tabular}{lcccc}
\hline Protein & Wt & Score & Tsc1 ${ }^{\text {GFAPCKO }}$ & Score \\
\hline EGF & ALL & + & ALL & +++ \\
EGFR & II-IV & +++ & II-IV & +++ \\
HGF & I-III & ++ & ALL & ++++ \\
HIF-1 $\alpha$ & ALL & 0 & ALL & ++ \\
C-Met & II-III & + & ALL & +++ \\
VEGF & II-III & ++ & II-V & ++++ \\
\hline
\end{tabular}

Semiquantitative scale $(0$, no staining; + , very few labeled cells;,$++>20$ labeled cells;,$+++>50$ labeled cells; ++++ , intense labeling across most cells in each ROI) to represent labeling density in each ROI. Roman numerical convention indicates expression of each growth factor within specified cortical layers; ALL, expression in all cortical layers. 

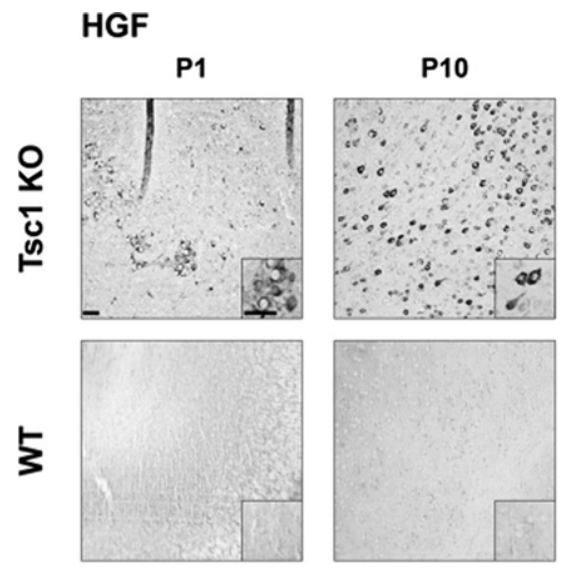

\section{c-Met}

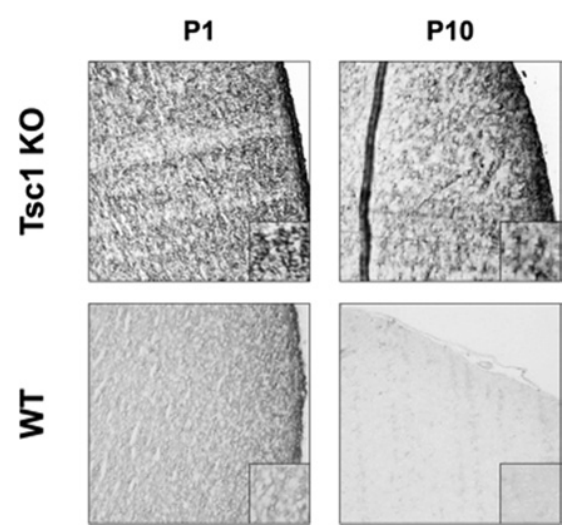

Figure 4. Enhanced expression of $\mathrm{HGF}$ and its receptor c-Met in TsC $1{ }^{\text {GFAP }}$ CKO (Tsc1 KO) mouse versus WT mouse cortex at postnatal (P) days 1 and 10; inset, original magnification, $\times 40$. Scale bars, $20 \mu \mathrm{m}$. Note expression of HGF in ectopic cell clusters depicted at P1 and throughout cortex at P10. In contrast, c-Met expression is more widespread across the cortex at both time points.

enhanced S6 protein phosphorylation in TsC1 ${ }^{\mathrm{GFAP}} \mathrm{CKO}$ mice compared with Wt control (Figure 1C). ${ }^{27}$ Immunolabeling of human TSC cortex also revealed an increase in phosphorylation of $\mathrm{S} 6$, relative to control cortex, with no corresponding increase in endogenous S6 (both phosphorylated and nonphosphorylated isoforms) expression overall (Supplemental Figure 1, see http://ajp.amjpathol. org). Increased S6 phosphorylation in tubers is consistent with previous findings in TSC and confirms mTOR hyperactivity in these lesions. ${ }^{28}$

\section{Increased Growth Factor mRNA Expression in Tsc ${ }^{\text {GFAP }}{ }^{2}$ KO Mice}

The expression of EGF, EGFR, HGF, c-Met, and VEGF mRNAs in cerebral cortex was increased at postnatal days 1 and 10 in TsC1 GFAPCKO mice compared with Wt mice (Figure 2). EGFR mRNA levels were increased 9.3fold and 7.1-fold at postnatal days 1 and 10, respectively, whereas EGF was increased 2.1-fold and 2.8-fold, HGF was increased 3.3-fold and fourfold, C-Met was increased threefold at both time points, and VEGF was increased 5.8-fold and 5.9-fold at postnatal days 1 and 10, respectively. However, Flt-1 mRNA expression in Tsc1 ${ }^{\text {GFAPCKO }}$ mice did not differ from that in Wt mice of either time point (data not shown). Of note, GFAP mRNA expression in the cortex was increased by 3.8-fold at postnatal day 1 and 6.4-fold at postnatal day 10 , consistent with previous reports that there is progressive increase in the number of astrocytes in the TSC1 ${ }^{\text {GFAPCKO mice. }}{ }^{26}$

\section{Increased Growth Factor Protein Expression in Tsc1 ${ }^{\text {GFAP }}$ CKO Mice}

Low levels of EGF, EGFR, HIF- $1 \alpha$, c-Met, and Flt- 1 expression were observed in Wt mice at postnatal days 1 and 10, whereas VEGF and HGF protein expression levels were higher at both time points. Because a substantial alteration of laminar architecture is not a feature of the TSC1 ${ }^{\text {GFAP }}$ CKO mouse cortex, we did not observe substantive changes in laminar expression of any of the growth factors or their receptors. Rather, our findings suggested enhanced expression in the expected distribution of each identified layer. For example, in the Wt mice, EGF was expressed at low levels across the cortical plate at both postnatal days 1 and 10, whereas in the TSC 1 GFAPCKO mouse, there was a global increase in EGF expression across all layers. HGF, C-Met, and VEGF exhibited an alteration in laminar expression profiles (Table 2) with enhanced expression in deeper cortical layers in
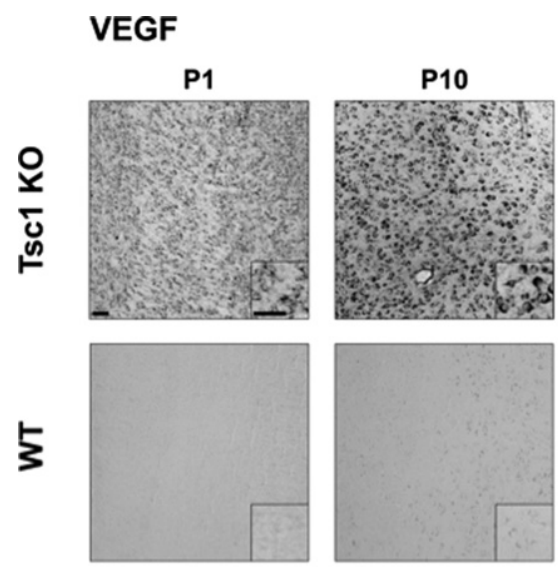

\section{HIF-1 $\alpha$}
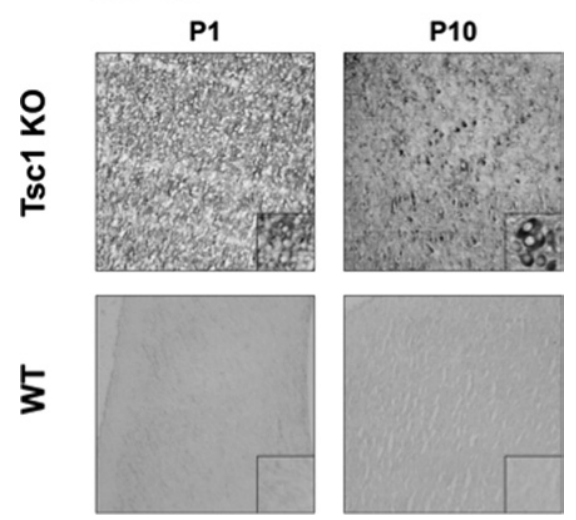

Figure 5. Enhanced expression of VEGF and HIF-1 $\alpha$ proteins in Tsc $1^{\mathrm{GFAP}} \mathrm{CKO}$ (Tsc1 KO) mouse versus WT mouse cortex at postnatal (P) days 1 and 10; inset, original magnification, $\times 40$; depicts VEGF or HIF- $1 \alpha$ labeling in individual cells or small clusters of cells. Scale bars, $20 \mu \mathrm{m}$. 
Table 3. Growth Factor and Growth Factor Receptor Expression in Tubers

\begin{tabular}{lcc}
\hline Protein & GCs, $\%$ & Distribution \\
\hline EGF & 68 & ALL \\
EGFR & 91 & ALL \\
Flt-1 & 11 & ALL \\
HGF & 74 & ALL \\
HIF-1 $\alpha$ & 42 & WM \\
C-Met & 71 & WM \\
VEGF & 83 & ALL \\
\hline
\end{tabular}

Table depicts percent of morphologically identified GCs expressing each protein marker, across all 10 tuber specimens. ALL, expression detected across all layers; WM, relative enrichment of labeled cells in subcortical white matter.

the TSC1 ${ }^{\text {GFAP }}$ CKO mouse. The expression of EGF, EGFR, HGF, C-Met, VEGF, and HIF- $1 \alpha$ proteins was increased at postnatal days 1 and 10 in TSC 1 GFAPCKO mice compared with Wt mice (Figures 3-5) in a profile that was similar although not identical to mRNA expression. However, similar to its corresponding mRNA, Flt-1 expression in TSC1 GFAPCKO mice did not differ from that in Wt mice at either time point (data not shown). By postnatal day 10, immunoreactive cells were identified in cerebral cortex across all six layers, and both neurons and astrocytes exhibited immunolabeling.

\section{Increased Growth Factor Protein in Human TSC Brain Tissue}

The mean maximal cell soma diameter of the GCs was $112.4 \mu \mathrm{m}$ (range, 104-125 $\mu \mathrm{m}$ ); thus, this parameter was used to generate cell counts for each individual protein marker. The expression profile for each protein marker was heterogeneous across the tubers and SEGAs. In some specimens, even when corrected for differing numbers of GCs across each case, there was robust expression of individual proteins, whereas in other specimens fewer numbers of cells were labeled (Table 3 ). For some proteins detailed below, there was enriched expression in GCs in deeper portions of the tuber (ie, within the
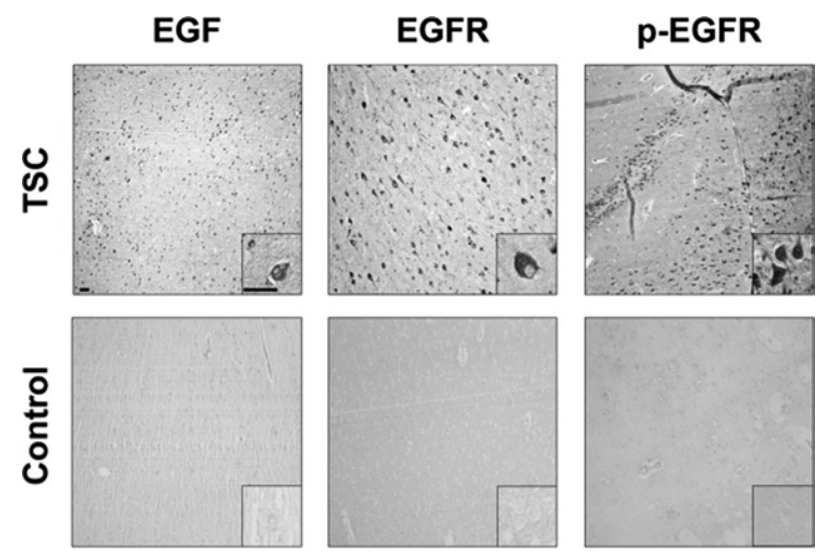

Figure 6. Increased EGF and EGFR (both phosphorylated and nonphosphorylated isoforms) expression in human TSC (tuber) versus control cortex; inset, original magnification, $\times 40$. Scale bars, $20 \mu \mathrm{m}$.

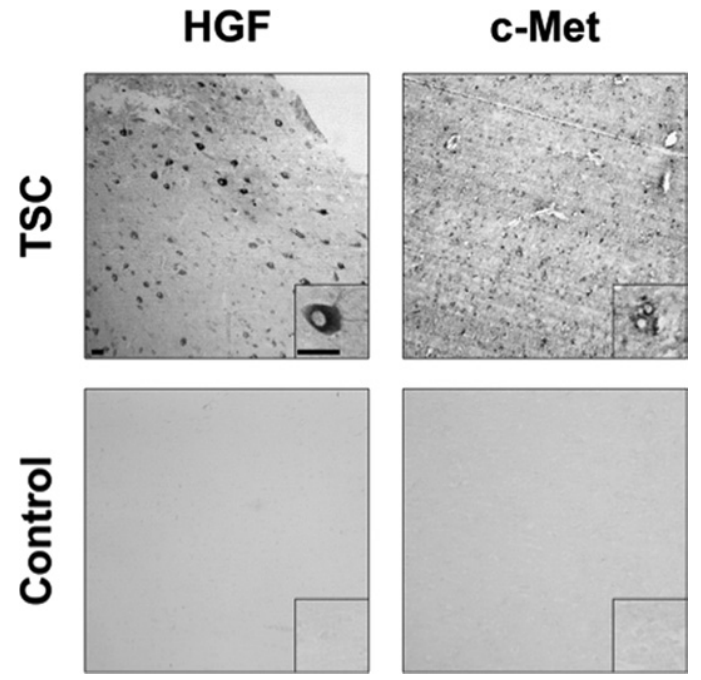

Figure 7. Increased HGF and c-Met expression in human TSC (tuber) versus control cortex; inset, original magnification, $\times 40$. Scale bars, $20 \mu \mathrm{m}$.

subcortical white matter) (Table 3), whereas for others, protein expression was observed in GCs throughout the thickness of the lesion (Table 3).

Immunohistochemical analysis demonstrated that EGF, EGFR, HGF, C-Met, VEGF, and HIF- $1 \alpha$ protein expression was markedly increased in tuber (Figures 6-9) and SEGA (Figure 10) specimens when compared with specimens of age-matched, postmortem, and non-TSC epilepsy cortex. Cellular expression of growth factor proteins was observed in GCs throughout the thickness of each tuber specimen, from pial surface to subcortical white matter. For example, expression of EGF and EGFR was primarily observed in GCs in tubers (Figure 6) and SEGAs (Figure 10). Expression of the phospho-isoform of EGFR was also enhanced in tubers compared with control cortex. The EGFRvIII variant protein isoform is frequently identified

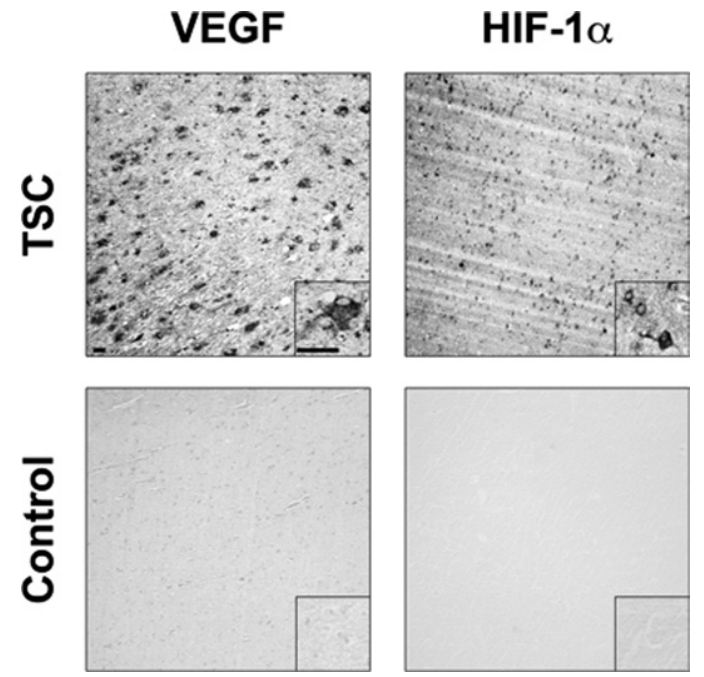

Figure 8. Increased VEGF and HIF-1 $\alpha$ expression in human (tuber) versus control cortex. Note VEGF expression in dysmorphic GCs; inset, original magnification, $\times 40$. Scale bars, $20 \mu \mathrm{m}$. 


\section{VEGF Expression}
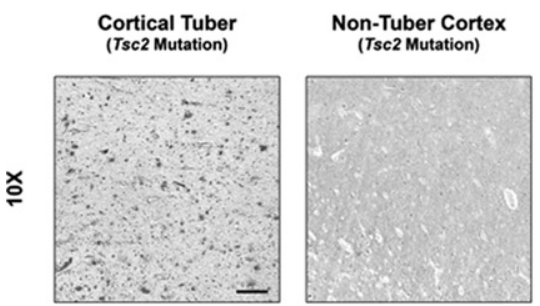

Non-TSC Epileptic Cortex (CASPR2 Mutation)
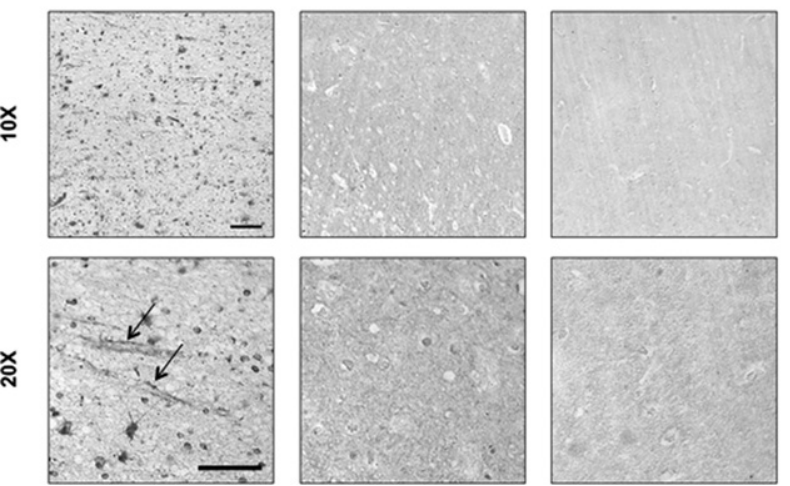

Figure 9. Increased expression of VEGF in a tuber specimen from a TSC patient, compared with nontuber cortex in the same TSC patient and with non-TSC epilepsy control cortex (top row, original magnification, $\times 10$ bottom row, original magnification, $\times 20$ ). In tuber cortex, there is robust cellular VEGF expression in both cells and blood vessels within the tissue (arrows). In nontuber cortex from the same TSC patient, there is overall less cellular VEGF expression than in tubers but more than in non-TSC epilepsy control cortex, in which there is little cellular VEGF expression. Scale bars, $100 \mu \mathrm{m}$.

as an overexpressed protein in certain types of astrocytomas and is linked to enhanced EGF signaling in these tumors. However, in contrast to native EGFR, there was no expression of EGFRvIII variant protein isoform in tubers or SEGAs (data not shown). HGF and c-Met expression was most marked in GCs and some of the surrounding dysmorphic neurons, within tubers (Figure 7) and SEGAs (Figure 10). VEGF was robustly expressed by GCs and capillary endothelial cells in tubers (Figure 8). In addition, the level of VEGF expression in nontuber cortex from TSC patients was increased relative to the level of VEGF expression in cortex from non-TSC epilepsy control patients (Figure 9). The distribution of HIF-1 $\alpha$ expression was similar to that of VEGF, although HIF- $1 \alpha$ expression was not as widespread in tubers and was often seen in small clusters of cells, especially in the subcortical white matter (Figure 8). In contrast, the levels of the VEGF receptor Flt-1 were not increased in either tubers or SEGAs (data not shown).

Growth factor and growth factor receptor expression in nontuber TSC cortex did not differ from non-TSC epilepsy control cortex, with the exception of VEGF. Specifically, rare GCs at the edge of the resection margin exhibited VEGF expression and appeared as a stark contrast to the surrounding tissue, and there were scattered VEGF-immunolabeled cells in the nontuber TSC cortex that were more frequent in number than in control non-TSC epilepsy cortex. These findings argued against a nonspecific effect of antiseizure medications, recurrent seizures, age, or postmortem interval on protein expression.

In addition, an important finding was that growth factor and growth factor receptor expression was not enhanced and appeared within these nodules to be similar to expression levels in control tissue (data not shown). These findings suggest that enhanced growth factor expression was indeed specific for tubers and SEGAs and hint toward a possible role of growth factors in the SEN to SEGA transformation.

\section{Discussion}

This is the first study, to our knowledge, to demonstrate enhanced EGF, EGFR, HGF, c-Met, and VEGF expression in association with loss of TSC1 in the mouse and with human TSC brain pathology. We were specifically interested in growth and angiogenic factors because of the documented enhancement of cell proliferation and angiogenesis in TSC-associated lesions, such as angiomyolipomas and SEGAs. The expression of EGF, EGFR, HGF, c-Met, and VEGF mRNAs was increased at postnatal

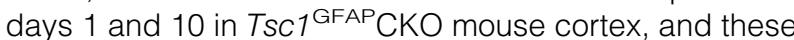
findings predicted similar changes in human TSC tubers and SEGAs. Increased mTOR signaling was demonstrated in TsC1 ${ }^{\text {GFAPC }}$ CKO mouse brain and human TSC cortical tubers and SEGAs, as evidenced by increased phosphorylation of S6 protein. We conclude that enhanced expression of EGF, EGFR, HGF, c-Met, and VEGF is linked at least in part to altered TSC1 or TSC2 function and aberrant mTOR signaling.

The Tsc1 ${ }^{\text {GFAP }}$ CKO mouse has been well characterized, and recent studies demonstrate that mTOR antagonism with rapamycin can ameliorate seizures and prevent premature death in this strain. ${ }^{27}$ Our study is the first, to our knowledge, to define the temporal pattern of mTOR activation, as evidenced by phosphorylation of S6 protein in the cortex of these animals. There is clearly a timedependent increase in S6 phosphorylation consistent with the ongoing effects of loss of Tsc1 after conditional inactivation. Indeed, a greater number of p-S6-immunolabeled cells were identified at postnatal day 10 than postnatal day 1 . We submit that changes in growth factor expression in the TsC ${ }^{\text {GFAP }}$ CKO mice did not reflect either seizures or behavioral alterations that appear later in this strain (approximately postnatal days 20 to 30) and rather represent direct effects of loss of Tsc1.

The effects of loss of TSC1 or TSC2 on expression of EGF, EGFR, HGF, c-Met, VEGF, and HIF- $1 \alpha$ in neural cells have not yet been studied, and we are the first to report changes in these growth factors and their recep-
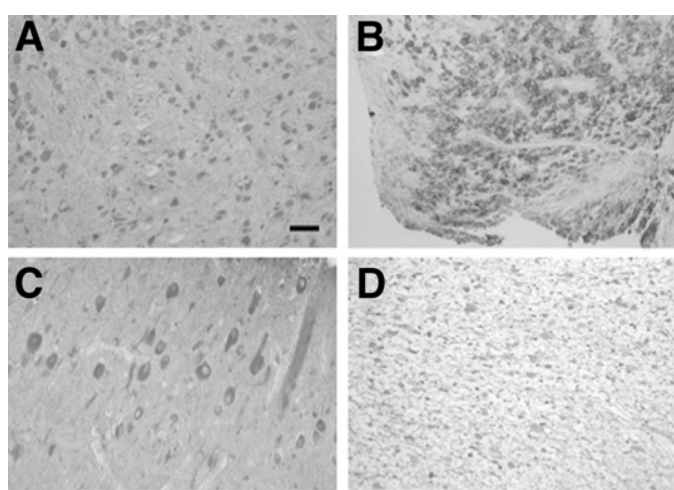

Figure 10. Expression of EGF (A), EGFR (B), HGF (C), and VEGF (D) in SEGAs. Scale bar, $100 \mu \mathrm{m}$. 
tors in TSC brain tissue with defined genotypes. Our findings suggest that altered growth factor expression may be a consequence of loss of either TSC1 or TSC2. EGFR plays a pivotal role in regulating astrocyte proliferation during development and has been implicated in aberrant cell growth control in malignant gliomas. EGFR has been localized to radial glial cells in the embryonic ventricular zone and is a marker for those that differentiate into astrocytes. Both HGF and its tyrosine kinase receptor c-Met are expressed by stem cells in the subventricular zone, ${ }^{29}$ and in view of the prior demonstration of stem cell markers such as nestin in tubers, ${ }^{30}$ these growth factors could also serve to characterize tubers as phenotypically immature. Like EGFR, HGF and c-Met are expressed in human gliomas and are believed to function in cell proliferation and angiogenesis (for review see Abounader and Laterra ${ }^{29}$ ). Functional blockade of EGFR and c-Met inhibits tumor growth. Prior studies have suggested that enhanced HGF expression in proliferating hepatocytes is mTOR dependent. ${ }^{31}$ Changes in EGF, EGFR, HGF, and c-Met expression were identified in the setting of diminished TSC1 or TSC2 function (ie, the TSC1GFAPCKO mouse or human tuber or SEGA specimens) but not in surrounding nontuber cortex and not within SENs, suggesting that complete TSC1 or TSC2 inactivation and commensurate hyperactivation of the mTOR pathway may be necessary to induce these effects.

VEGF expression is directly modulated by TSC1 and TSC2 via both mTOR-dependent and mTOR-independent pathways in vitro. ${ }^{25}$ Thus, among the growth factor genes assayed, alterations in VEGF mRNA and protein levels may be most sensitive to loss of TSC1 or TSC2. ${ }^{22}$ High levels of VEGF mRNA expression have been reported in angiomyolipomas and in serum from human TSC patients. ${ }^{23,32}$ VEGF expression was enhanced after TsC1 knockout in vivo in mice, and we observed enhanced VEGF expression in human tubers and SEGAs, as well as in perituberal cortex.

Increased mTOR signaling, corresponding to loss of TSC1/TSC2 inhibition, has been demonstrated specifically in SEGAs ${ }^{33}$ and tubers. ${ }^{28}$ Thus, the findings in human TSC tissue specimens of increased expression of EGF, HGF, VEGF, and their receptors primarily within these focal areas of mTOR cascade dysregulation suggest that reduction of TSC1 or TSC2 could lead to increased EGF, HGF, or VEGF levels via mTOR. Indeed, our results support previous observations in mouse kidney cells and fibroblasts demonstrating enhanced VEGF detected in either Tsc2 heterozygous $(+/-)$ or null $(-/-)$ cells. ${ }^{22}$

These results provide a link between aberrant mTOR cascade activation and expression of growth factors that could be targeted for future therapeutic trials in TSC. EGFR, HGF, and VEGF can be blocked with specific antagonists, perhaps in association with rapamycin, as a strategy to reduce the size of tubers or SEGAs in TSC and diminish the potential toxicity of any one therapeutic compound.

\section{References}

1. Consortium TECTS: Identification and characterization of the tuberous sclerosis gene on chromosome 16. Cell 1993, 75:1305-1315

2. van Slegtenhorst $M$, de Hoogt $R$, Hermans $C$, Nellist $M$, Janssen $B$, Verhoef S, Lindhout D, van den Ouweland A, Halley D, Young J, Burley M, Jeremiah S, Woodward K, Nahmias J, Fox M, Ekong R, Osborne J, Wolfe J, Povey S, Snell RG, Cheadle JP, Jones AC Tachataki M, Ravine D, Sampson JR, Reeve MP, Richardson P, Wilmer F, Munro C, Hawkins TL, Sepp T, Ali JB, Ward S, Green AJ, Yates JR, Kwiatkowska J, Henske EP, Short MP, Haines JH, Jozwiak S, Kwiatkowski DJ: Identification of the tuberous sclerosis gene TSC1 on chromosome 9q34. Science 1997, 277:805-808

3. Bolton PF, Park RJ, Higgins JN, Griffiths PD, Pickles A: Neuro-epileptic determinants of autism spectrum disorders in tuberous sclerosis complex. Brain 2002, 125:1247-1255

4. Gomez MR, Sampson JR, Whittemore VH: Tuberous Sclerosis Complex. New York, Oxford University Press, 1999, xxi, p 340.

5. Koh S, Jayakar P, Dunoyer C, Whiting SE, Resnick TJ, Alvarez LA Morrison G, Ragheb J, Prats A, Dean P, Gilman J, Duchowny MS Epilepsy surgery in children with tuberous sclerosis complex: presurgical evaluation and outcome. Epilepsia 2000, 41:1206-1213

6. Sparagana SP, Roach ES: Tuberous sclerosis complex. Curr Opin Neurol 2000, 13:115-119

7. Sharma M, Ralte A, Arora R, Santosh V, Shankar SK, Sarkar C: Subependymal giant cell astrocytoma: a clinicopathological study of 23 cases with special emphasis on proliferative markers and expression of p53 and retinoblastoma gene proteins. Pathology 2004, 36 $139-144$

8. Gyure KA, Prayson RA: Subependymal giant cell astrocytoma: a clinicopathologic study with HMB45 and MIB-1 immunohistochemical analysis. Mod Pathol 1997, 10:313-317

9. Kim SK, Wang KC, Cho BK, Jung HW, Lee YJ, Chung YS, Lee JY, Park SH, Kim YM, Choe G, Chi JG: Biological behavior and tumorigenesis of subependymal giant cell astrocytomas. J Neurooncol 2001, 52:217-225

10. Lopes MB, Altermatt HJ, Scheithauer BW, Shepherd CW, VandenBerg SR: Immunohistochemical characterization of subependymal giant cell astrocytomas. Acta Neuropathol 1996, 91:368-375

11. Mizuguchi M, Takashima S: Neuropathology of tuberous sclerosis. Brain Dev 2001, 23:508-515

12. Lee A, Maldonado M, Baybis M, Walsh CA, Scheithauer B, Yeung R Parent J, Weiner HL, Crino PB: Markers of cellular proliferation are expressed in cortical tubers. Ann Neurol 2003, 53:668-673

13. Ess KC, Kamp CA, Tu BP, Gutmann DH: Developmental origin of subependymal giant cell astrocytoma in tuberous sclerosis complex. Neurology 2005, 64:1446-1449

14. Fingar DC, Blenis J: Target of rapamycin (TOR): an integrator of nutrient and growth factor signals and coordinator of cell growth and cell cycle progression. Oncogene 2004, 23:3151-3171

15. Kyin R, Hua $Y$, Baybis M, Scheithauer B, Kolson D, Uhlmann E, Gutmann D, Crino PB: Differential cellular expression of neurotrophins in cortical tubers of the tuberous sclerosis complex. Am J Pathol 2001, 159:1541-1554

16. Harrington LS, Findlay GM, Gray A, Tolkacheva T, Wigfield S, Rebholz H, Barnett J, Leslie NR, Cheng S, Shepherd PR, Gout I, Downes CP, Lamb RF: The TSC1-2 tumor suppressor controls insulin-PI3K signaling via regulation of IRS proteins. J Cell Biol 2004, 166:213-223

17. Shah OJ, Wang Z, Hunter T: Inappropriate activation of the TSC/ Rheb/mTOR/S6K cassette induces IRS1/2 depletion, insulin resistance, and cell survival deficiencies. Curr Biol 2004, 14:1650-1656

18. Shah OJ, Hunter T: Turnover of the active fraction of IRS1 involves raptor-mTOR- and S6K1-dependent serine phosphorylation in cell culture models of tuberous sclerosis. Mol Cell Biol 2006, 26:64256434

19. Brugarolas J, Kaelin WG Jr: Dysregulation of HIF and VEGF is a unifying feature of the familial hamartoma syndromes. Cancer Cell 2004, 6:7-10

20. Lesma E, Grande V, Ancona S, Carelli S, Di Giulio AM, Gorio A Anti-EGFR antibody efficiently and specifically inhibits human TSC2-/- smooth muscle cell proliferation: possible treatment options for TSC and LAM. PLoS One 2008, 3:e3558 
21. Nguyen-Vu PA, Fackler I, Rust A, DeClue JE, Sander CA, Volkenandt M, Flaig M, Yeung RS, Wienecke R: Loss of tuberin, the tuberoussclerosis-complex-2 gene product is associated with angiogenesis. J Cutan Pathol 2001, 28:470-475

22. El-Hashemite N, Walker V, Zhang H, Kwiatkowski DJ: Loss of Tsc 1 or Tsc2 induces vascular endothelial growth factor production through mammalian target of rapamycin. Cancer Res 2003, 63: 5173-5177

23. Arbiser JL, Brat D, Hunter S, D'Armiento J, Henske EP, Arbiser ZK, Bai X, Goldberg G, Cohen C, Weiss SW: Tuberous sclerosis-associated lesions of the kidney, brain, and skin are angiogenic neoplasms. J Am Acad Dermatol 2002, 46:376-380

24. McCormack FX: Lymphangioleiomyomatosis: a clinical update. Chest 2008, 133:507-516

25. Brugarolas JB, Vazquez F, Reddy A, Sellers WR, Kaelin WG Jr: TSC2 regulates VEGF through mTOR-dependent and -independent pathways. Cancer Cell 2003, 4:147-158

26. Uhlmann EJ, Wong M, Baldwin RL, Bajenaru ML, Onda H, Kwiatkowski DJ, Yamada K, Gutmann DH: Astrocyte-specific TSC1 conditional knockout mice exhibit abnormal neuronal organization and seizures. Ann Neurol 2002, 52:285-296
27. Zeng LH, Xu L, Gutmann DH, Wong M: Rapamycin prevents epilepsy in a mouse model of tuberous sclerosis complex. Ann Neurol 2008 63:444-453

28. Baybis M, Yu J, Lee A, Golden JA, Weiner H, McKhann G, 2nd Aronica E, Crino PB: mTOR cascade activation distinguishes tubers from focal cortical dysplasia. Ann Neurol 2004, 56:478-487

29. Abounader R, Laterra J: Scatter factor/hepatocyte growth factor in brain tumor growth and angiogenesis. Neuro Oncol 2005, 7:436-451

30. Crino PB, Trojanowski JQ, Dichter MA, Eberwine J: Embryonic neuronal markers in tuberous sclerosis: single-cell molecular pathology. Proc Natl Acad Sci U S A 1996, 93:14152-14157

31. Palmes D, Zibert A, Budny T, Bahde R, Minin E, Kebschull L, Holzen J, Schmidt H, Spiegel HU: Impact of rapamycin on liver regeneration. Virchows Arch 2008, 452:545-557

32. Young LR, Inoue Y, McCormack FX: Diagnostic potential of serum VEGF-D for lymphangioleiomyomatosis. N Engl J Med 2008, 358 199-200

33. Chan JA, Zhang $H$, Roberts PS, Jozwiak S, Wieslawa G, LewinKowalik J, Kotulska K, Kwiatkowski DJ: Pathogenesis of tuberous sclerosis subependymal giant cell astrocytomas: biallelic inactivation of TSC1 or TSC2 leads to mTOR activation. J Neuropathol Exp Neurol 2004, 63:1236-1242 